\title{
Influence of Early and Late Zoledronic Acid Administration on Vertebral Structure and Strength in Ovariectomized Rats
}

\author{
Julienne E. M. Brouwers · Bert van Rietbergen • \\ Mary L. Bouxsein
}

Received: 16 June 2008/ Accepted: 2 July 2008/Published online: 19 August 2008

(c) The Author(s) 2008. This article is published with open access at Springerlink.com

\begin{abstract}
An annual infusion of zoledronic acid (ZOL) reduces fracture risk in osteoporotic patients. Previously, we showed that a single ZOL injection inhibited changes in bone microstructure and strength in rat tibiae after ovariectomy. Here, we determined the effects of a single ZOL injection as preventive and restorative treatment on the bone microstructure and strength in lumbar and caudal vertebrae of ovariectomized (OVX) rats. Twenty-nine female 35-weekold Wistar rats were divided into four groups: SHAM-OVX $(n=9)$, OVX $(n=5)$, OVX and early ZOL $(n=8)$, and OVX and late ZOL $(n=7)$. ZOL was given once $(20 \mu \mathrm{g} / \mathrm{kg}$ body weight s.c.) at OVX in the early ZOL group and 8 weeks later in the late $\mathrm{ZOL}$ group; rats were killed 16 weeks after OVX. Trabecular and cortical bone microarchitecture were measured in lumbar (L3) and caudal (Cd6) vertebrae using micro-computed tomography, and compressive mechanical properties were determined in L3 vertebrae. Compared to SHAM-OVX, OVX rats had significantly lower BV/TV; SMI, Tb.N, Tb.Sp, and Conn.D tended to be deteriorated in lumbar vertebrae, while both ZOL groups did not differ from the SHAM-OVX group. Both ZOL groups had significantly higher BV/TV than
\end{abstract}

\footnotetext{
J. E. M. Brouwers

Eindhoven University of Technology, Den Dolech 2,

5600 MB Eindhoven, The Netherlands

e-mail: j.e.m.brouwers@tue.nl

B. van Rietbergen

Eindhoven University of Technology, Eindhoven,

The Netherlands

J. E. M. Brouwers · M. L. Bouxsein ( $₫)$

Orthopedic Biomechanics Laboratory, Beth Israel Deaconess

Medical Center and Harvard Medical School,

330 Brookline Avenue, Boston, MA 02215, USA

e-mail: mbouxsei@bidmc.harvard.edu
}

OVX; the early ZOL group also had significantly lower SMI and higher Tb.Th. OVX tended to decrease mechanical properties, while early and late ZOL treatment inhibited OVX-induced degeneration. Neither OVX nor ZOL induced changes in the trabecular microarchitecture of caudal vertebrae. In summary, in adult rats a single ZOL injection inhibited OVX-induced changes in lumbar vertebral bone microarchitecture and strength.

Keywords Bisphosphonate - Bone architecture/ structure $\cdot$ Osteoporosis $\cdot$ Animal model

Bisphosphonates inhibit bone resorption and are commonly used to treat osteoporotic patients. They slow the process of bone loss and reduce the risk of fracture by maintaining bone mass and microstructure [1-3]. Zoledronic acid (ZOL) is a potent bisphosphonate that has recently been shown to significantly reduce fracture risk in osteoporotic patients who received once-yearly doses $[4,5]$. As the number and depth of in vivo analyses in patients are limited, animal research is ongoing to further elucidate the effects of ZOL on skeletal fragility [6-9]. Previously, we showed in a longitudinal study of adult rats that a single injection of $\mathrm{ZOL}$ at the time of ovariectomy (OVX) inhibited OVX-induced deterioration of bone microstructure and strength in the proximal tibiae for up to 16 weeks [10]. Furthermore, a single injection of ZOL 8 weeks after OVX inhibited further deterioration of the bone microstructure and strength in rat tibiae compared to untreated OVX rats. Other studies have shown that weekly injections of ZOL inhibit changes in bone microstructure and strength in rat vertebrae after OVX $[8,9]$. However, it is not known to what extent a single dose of ZOL is able to inhibit and restore OVX-induced loss of bone mass, structure, and strength in rat vertebrae. 
Most studies in rats conducted to determine bone changes induced by OVX and/or the efficacy of pharmacological intervention evaluate skeletal sites with red (hematopoietic) marrow, such as the proximal tibia and lumbar vertebra. Yet, bone sites with yellow (fatty) marrow, such as the distal tibia and caudal vertebra, are known to have different cell composition from red marrow bone sites and may respond differently to estrogen depletion [11]. Also, caudal vertebrae in rats are subject to a different loading environment from other vertebrae, which may also affect the response to OVX and/or drug intervention.

The goal of this study was to determine the effects of a single ZOL injection as a preventive and restorative treatment on bone microstructure and strength in lumbar vertebrae of OVX rats. Additionally, we assessed whether lumber and caudal vertebrae respond similarly to OVX and/or ZOL treatment in terms of bone microarchitecture.

\section{Methods}

Twenty-nine female 35-week-old Wistar rats were used from a previous study [10]. In brief, rats were divided into four groups based on weight: SHAM-OVX $(n=9)$, OVX $(n=5)$, OVX and early ZOL $(n=8)$, and OVX and late ZOL $(n=7)$. Sample size, calculated for the previously conducted longitudinal study, was nine per group. However, the number of animals was lowered and differed between groups due to nonsuccessful OVX, determined after death based on uterine weight. All rats underwent OVX at week 0; the SHAM-OVX group underwent a sham operation. The early ZOL group received ZOL at a single dose of $20 \mu \mathrm{g} / \mathrm{kg}$ body weight s.c. at the time of OVX. The dose was chosen based on a dose-response study in rats, in which $20 \mu \mathrm{g} / \mathrm{kg}$ body weight was found to be most effective [12]. The late ZOL group received the same single dose 8 weeks after OVX. Rats were killed 16 weeks after OVX by exsanguination.

After death, whole lumbar (L3) and caudal (Cd6) vertebrae were dissected, soaked in $0.9 \%$ saline solution gauze, and frozen at $-20^{\circ} \mathrm{C}$. This study was approved by the Animals Ethics Committee of the University of Maastricht, the Netherlands. ZOL was kindly provided as the disodium salt hydrate by Novartis Pharma (Basel, Switzerland) and dissolved in a saline vehicle prior to injection.

\section{Assessment of Vertebral Microarchitecture}

L3 and Cd6 vertebrae were thawed to room temperature and scanned using a high-resolution desktop microtomographic imaging system (microCT40; Scanco Medical, Bruetisellen, Switzerland) at an isotropic voxel size of $16 \mu \mathrm{m}(55 \mathrm{kV}, 145 \mu \mathrm{A}, 500$ projections per 180 degrees,
$200 \mathrm{~ms}$ integration time $)$ and $12 \mu \mathrm{m}(55 \mathrm{kV}, 145 \mu \mathrm{A}, 500$ projections per 180 degrees, $200 \mathrm{~ms}$ integration time), respectively. A smaller voxel size was chosen for the caudal vertebrae as their size, and therefore volume of interest, is smaller than lumbar vertebrae. After scanning, samples were frozen again until mechanical testing. Images were subjected to a gaussian filter and binarized ( sigma $=0.8$, support $=1$, threshold $=32 \%$ of maximal gray-scale value) to separate bone from background [13].

For the L3 vertebrae, the trabecular region was manually selected starting 10 slices below the cranial growth plate and ending 10 slices above the caudal growth plate, resulting in a trabecular region of approximately $5 \mathrm{~mm}$ in the axial direction. From this region, six bone structural parameters (bone volume fraction [BV/TV], connectivity density [Conn.D], structure model index [SMI], trabecular number [Tb.N], trabecular thickness [Tb.Th], and trabecular separation [Tb.Sp]) were automatically determined. Cortical bone was semiautomatically selected from computed tomographic (CT) scans, using the same set of slices as used when selecting the trabecular bone, from which the average cortical thickness was determined. Because the caudal vertebrae had been shown to be symmetrical in the cranial-caudal direction, the top half of trabecular bone was manually selected in the CT scans of the Cd6 vertebrae, starting 10 slices below the cranial growth plate. From this region, the same structural parameters were determined as in the L3 vertebrae.

\section{Static Vertebral Compression Tests}

All L3 vertebrae were thawed to room temperature prior to mechanical testing. To achieve planoparallel ends, vertebrae were fixed in a custom-made jig. A double-blade, lowspeed diamond saw (IsoMet; Buehler, Lake Bluff, IL) was used under constant saline irrigation to remove cranial and caudal ends including the growth plate, resulting in a vertebral height of about $4.2 \mathrm{~mm}$. A single-blade, wafering, low-speed diamond saw was used under constant saline irrigation to remove all posterior pedicles and processes. Anterior elements were clipped off, resulting in an isolated vertebral body. After sawing, the exact vertebral height was measured using a caliper. Vertebrae were kept frozen in a $0.9 \%$ saline solution until mechanical testing.

After thawing, vertebrae were compressed at a constant displacement rate $(3 \mathrm{~mm} / \mathrm{min})$ until failure (Synergie 100 ; MTS Systems, Eden Prairie, MN). Displacement was measured from the actuator displacement transducer of the testing machine and corrected for stiffness of the testing system. From the force-displacement curve, the following mechanical parameters were determined: (1) stiffness, calculated as the slope in the linear region between $40 \%$ and $80 \%$ of the maximum force; (2) ultimate load, defined 
as the maximum load; (3) displacement at ultimate load; and (4) energy to ultimate load, defined as the area under the curve until ultimate load.

\section{Data Analysis}

All parameters were compared between groups using analysis of variance (ANOVA) with Bonferroni's post-hoc test comparing all groups with each other. A two-way ANOVA was done to determine whether the response to OVX and ZOL was different between the L3 and Cd6 vertebrae. $P<0.05$ was considered significant.

\section{Results}

Effects of OVX and ZOL on Lumbar Vertebral Microarchitecture

Compared to SHAM, OVX rats had a significantly lower BV/TV in L3 vertebrae (Fig. 1). SMI, Tb.N, Tb.Sp, and Conn.D were also substantially deteriorated in the OVX group; however, these differences did not reach significance. Both ZOL groups had significantly higher BV/TV than OVX; the early ZOL group also had significantly lower SMI and higher Tb.Th compared to OVX. Trabecular microarchitecture did not differ between the SHAMOVX and ZOL-treated groups, except for Tb.Th, which was significantly higher in the early ZOL group than in the SHAM-OVX group. Trabecular architecture did not differ between the early and late ZOL groups. Cortical thickness did not differ between all groups.
Effects of OVX and ZOL on Vertebral Compressive Properties

Compressive stiffness and ultimate load were lower in OVX compared to other groups, though this did not reach statistical significance (Fig. 2). There was a trend for both the early and late ZOL groups to have slightly higher stiffness and failure load than the SHAM-OVX group. Displacement at ultimate load was similar in OVX and SHAM groups, while it tended to be lower in both ZOL groups. No differences were observed in energy to failure.

Comparison Between Response to OVX and ZOL in Lumbar and Caudal Vertebrae

In the Cd6 vertebrae, trabecular architecture did not differ between groups (Fig. 3). The response to OVX was significantly different between caudal and lumbar vertebrae for BV/TV and SMI $\left(P_{\text {interaction }}=0.010\right.$ and 0.012 , respectively). ZOL treatment did not alter bone microarchitecture, which may be expected since OVX did not lead to trabecular deterioration in the caudal vertebrae.

\section{Discussion}

In this study, we found that (1) a single ZOL injection inhibited deterioration of trabecular bone microarchitecture and strength in lumbar vertebrae of OVX rats, (2) neither OVX nor ZOL treatment influenced microarchitecture in caudal vertebrae, and (3) differences between early and late ZOL treatment were not detectable by end-of-study measurements.
Fig. 1 Effect of OVX and ZOL treatment on trabecular microarchitecture of lumbar (L3) vertebrae (mean $\pm \mathrm{SD}$ ). (a) Significantly different from OVX; (b) significantly different from OVX-SHAM
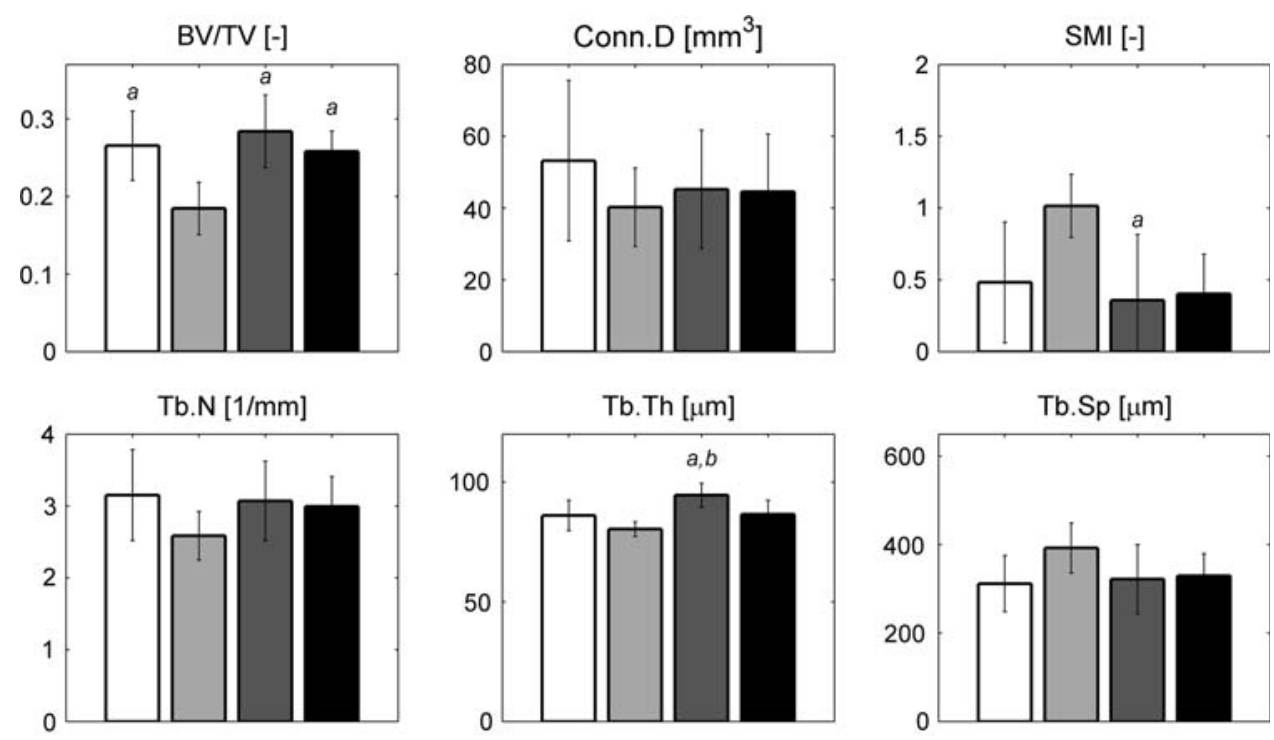

SHAM-OVX

early ZOL

late ZOL 
Fig. 2 Effect of OVX and ZOL treatment on quasi-static compressive mechanical properties of lumbar vertebrae $($ mean $\pm \mathrm{SD})$
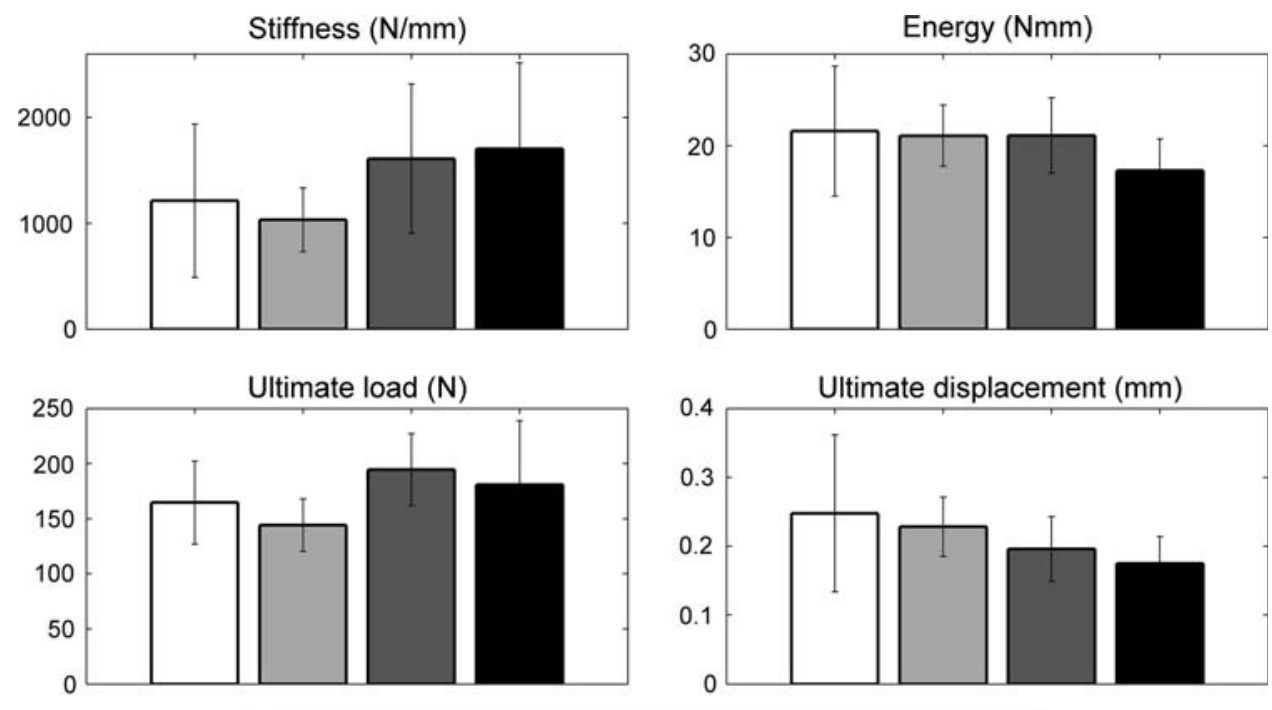

SHAM-OVX $\square$ OVX $\square$ early ZOL

late ZOL

Fig. 3 Effect of OVX and ZOL treatment on trabecular microarchitecture of caudal (Cd6) vertebra (mean $\pm \mathrm{SD})$
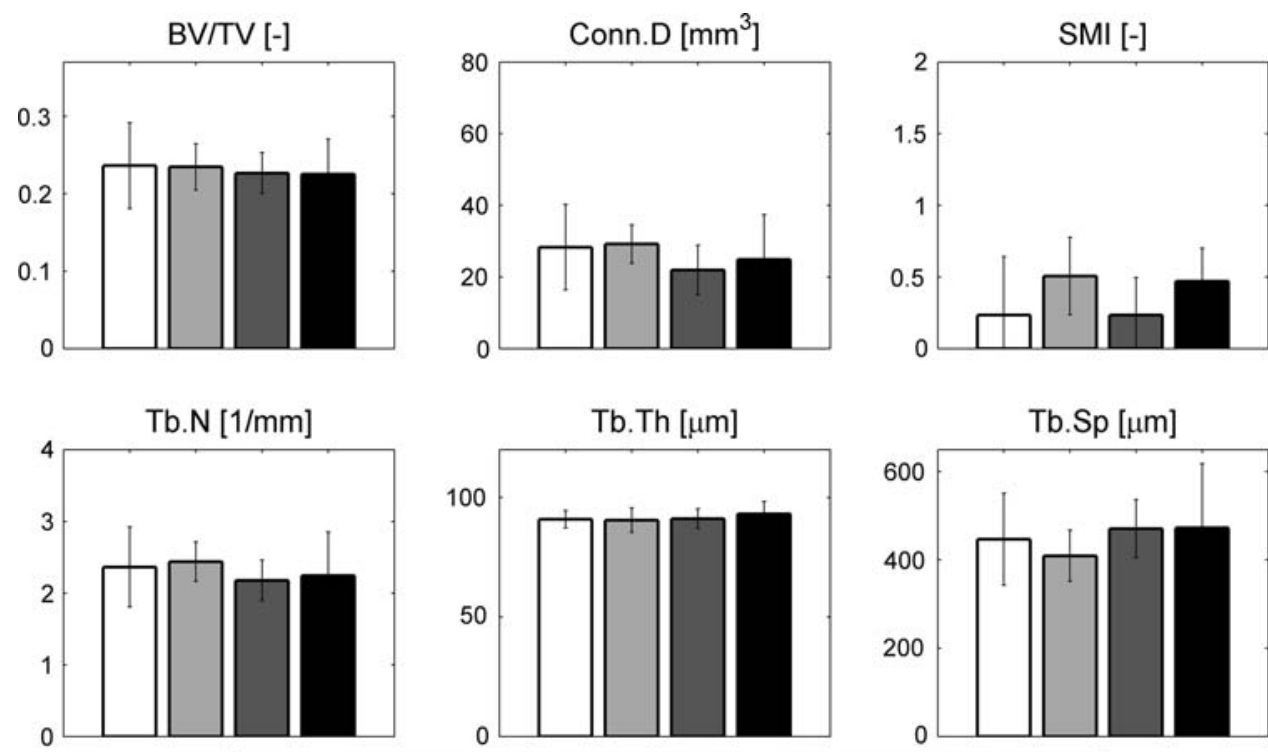

Tb.Th $[\mu \mathrm{m}]$
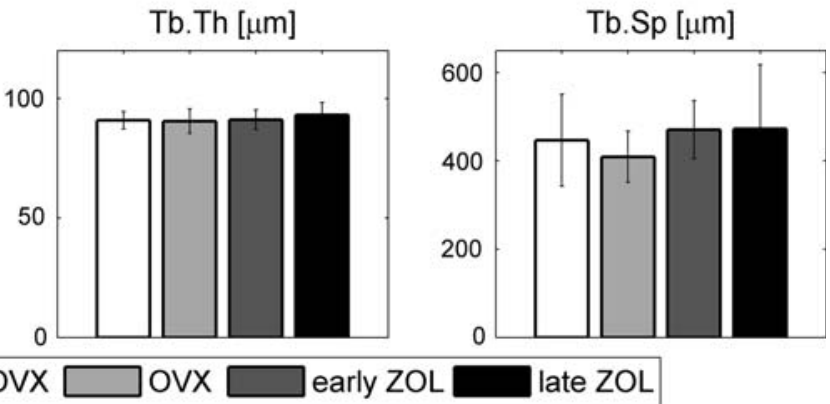

Previously, it was shown that 1 year of weekly injections of ZOL inhibited OVX-induced changes in bone microstructure and bone strength of femora and lumbar vertebrae of rats [7-9]. Also, in the same rats as used in this study, a single injection of ZOL prevented deterioration of bone microstructure and strength in tibiae of OVX rats for 16 weeks and a single injection at 8 weeks after OVX prevented further deterioration of structure and strength [10]. Additionally, in a dose-response study, it was shown that a single injection can inhibit structural changes seen after OVX in the tibia and retain mechanical properties in the vertebra [12]. In this study, we determined that a single injection of ZOL can inhibit changes in bone microstructure and strength in vertebrae. Vertebrae present a clinically relevant anatomical site for the treatment of osteoporosis. As such, our study includes novel and relevant information on the effects of a single ZOL injection on trabecular bone microarchitecture and cortical thickness in lumbar as well as in caudal vertebrae. The pattern in bone volume fraction between the four groups in the lumbar vertebrae was exactly the same as that seen in the proximal tibiae [10]. Also, a significant correlation was found between all individual values for bone volume fraction of the lumbar vertebrae and those of the tibiae $\left(r^{2}=0.4, P<0.001\right.$; data not shown). This indicates that the response to OVX and ZOL was similar in the tibiae and the lumbar vertebrae.

Mechanical properties of lumbar vertebrae were reduced in OVX vs. SHAM-OVX rats, while treatment with ZOL 
inhibited these losses. Both stiffness and ultimate load in the ZOL-treated groups were similar to values in the SHAM-OVX group. Ultimate displacement tended to be lower in both ZOL groups compared to the SHAM-OVX and OVX groups, which could be an indication of a slightly more brittle tissue due to increased mineralization, although in a recent study no effect of ZOL on mineral crystal size was determined in ZOL-treated, OVX rats [14]. In general, we conclude that both early and late ZOL treatment resulted in overall, normal mechanical properties.

Unique aspects of our study were that the effect of OVX on caudal vertebrae was analyzed using micro-CT and that the effect of ZOL treatment on OVX rats was studied in caudal vertebrae. In contrast to the results from lumbar vertebrae, trabecular architecture in caudal vertebrae did not change in response to OVX and ZOL. Our results concur with previous reports on nonresponsiveness to OVX in caudal vertebrae [11, 15-17]. It has been suggested that differences in marrow composition between lumbar and caudal vertebrae play a role in responsiveness to OVX [11]. Cancellous bone turnover in yellow marrow, such as in the caudal vertebrae, is lower than in red marrow sites, such as in the lumbar vertebra, as evidenced by decreased osteoclast surface, osteoclast number, mineral apposition rate, and bone formation rate [11]. These differences may be related to the cellular composition and vascularity of bone marrow. Also, it has been shown that the response to parathyroid hormone is different between caudal and lumbar vertebrae [15]. Altogether, this indicates that the responses to OVX and to different drug treatments are different in the caudal and lumbar vertebrae, rendering the caudal vertebra less interesting as a therapeutic testing site.

Differences between early and late ZOL treatment were not detectable by end-of-study measurements, though early ZOL treatment had a slightly better effect on bone morphology compared to late ZOL treatment. The late ZOL group received the ZOL injection 8 weeks after OVX. It is known that 8 weeks after OVX bone microarchitecture has significantly deteriorated in the rat vertebra [18]. As bone volume fraction was similar in the early and late ZOL groups, we postulate that after ZOL treatment bone volume fraction somewhat increased, as seen in the tibiae as well [10], probably due to the filling of resorption cavities [19].

This study had several limitations. The tibiae of rats were previously analyzed by in vivo micro-CT, and the groups were found to have slightly different baseline values. Since in this study only end-point measurements of the vertebrae were performed, it is possible that there was also a small difference in baseline values in the vertebrae of these rats. Previously, however, we showed in the longitudinal study that these differences did not influence overall trends between groups in the tibiae; therefore, it was thought that overall trends in the vertebrae would be similar as well [10]. Also, the rats were scanned several times while living, which could have led to radiation damage. However, we showed previously that the design of the rat holder was such that only the scanned leg was exposed to radiation and that no radiation damage was found in the scanned leg after eight weekly scans [20]. Therefore, any radiation damage can be ruled out.

Another limitation to this study was the fact that we were unable to detect significant deterioration of mechanical properties resulting from OVX as well as differences between early and late ZOL treatment by endof-study measurements. This lack of significant effect may relate to the fact that the study was designed for follow-up measurements, which makes the number of samples needed lower than desirable for the present study. For these cross-sectional measurements in the vertebrae, sample sizes were relatively low, reducing the power of the study to detect significant differences. However, it should be noted that our primary question was whether a single injection can prevent OVX-induced bone loss. We were able to answer this question with adequate power and statistical significance. In the current study, also a nonsignificant difference of $10 \%$ between the early and late ZOL groups in bone volume fraction was found. It can be argued whether a study should be designed to detect a small difference of $10 \%$ and whether this is scientifically relevant. A power study indicated that with the sample size and variance seen in bone volume fraction of lumbar vertebrae and a generally assumed power of $80 \%$ and alpha of 0.05 , a difference of $20 \%$ in mean between the early and late ZOL groups would have been detected. Thus, although the study did not have the power to detect a small difference of $10 \%$, it was adequately powered to answer the primary question and to answer secondary questions if the differences were large enough to be clinically relevant (i.e., on the order of 20\%). It should also be noted that in previous studies on the effects of weekly ZOL injections on mechanical properties, group sample sizes were 16-20, offering a higher power to detect differences [7, 9]. Therefore, further studies with a larger sample size are needed to distinguish between the effects of early and late ZOL treatments on vertebral trabecular microarchitecture and strength.

In conclusion, we showed that a single injection of ZOL inhibits deterioration of bone microstructure and strength in lumbar vertebrae of OVX rats, demonstrating the potent, long-term antiresorptive effect of this therapy. These data corroborate data from human iliac biopsies taken from osteoporotic patients after 3 years of ZOL treatment [21]. Altogether, these positive effects on trabecular microarchitecture and strength likely contribute to the antifracture efficacy of ZOL. 
Acknowledgements This work was funded by the Netherlands Organization for Scientific Research, Prins Bernard Cultuurfonds, and a scholarship from VSBfonds. We thank John Muller for technical advice and assistance with mechanical testing.

Open Access This article is distributed under the terms of the Creative Commons Attribution Noncommercial License which permits any noncommercial use, distribution, and reproduction in any medium, provided the original author(s) and source are credited.

\section{References}

1. Borah B, Dufresne TE, Chmielewski PA, Johnson TD, Chines A, Manhart MD (2004) Risedronate preserves bone architecture in postmenopausal women with osteoporosis as measured by threedimensional microcomputed tomography. Bone 34:736-746

2. Hordon LD, Itoda M, Shore PA, Shore RC, Heald M, Brown M, Kanis JA, Rodan GA, Aaron JE (2006) Preservation of thoracic spine microarchitecture by alendronate: comparison of histology and microCT. Bone 38:444-449

3. Russell RGG (2006) Ibandronate: pharmacology and preclinical studies. Bone 38:S7-S12

4. Lyles KW, Colon-Emeric CS, Magaziner JS, Adachi JD, Pieper CF, Mautalen C, Hyldstrup L, Recknor C, Nordsletten L, Moore KA, Lavecchia C, Zhang J, Mesenbrink P, Hodgson PK, Abrams K, Orloff JJ, Horowitz Z, Eriksen EF, Boonen S (2007) Zoledronic acid and clinical fractures and mortality after hip fracture. $\mathrm{N}$ Engl J Med 357:1799-1809

5. Black DM, Delmas PD, Eastell R, Reid IR, Boonen S, Cauley JA, Cosman F, Lakatos P, Leung PC, Man Z, Mautalen C, Mesenbrink P, Hu H, Caminis J, Tong K, Rosario-Jansen T, Krasnow J, Hue TF, Sellmeyer D, Eriksen EF, Cummings SR, HORIZON Pivotal Fracture Trial (2007) Once-yearly zoledronic acid for treatment of postmenopausal osteoporosis. N Engl J Med 356:1809-1822

6. Gasser JA, Green JR, Shen V, Ingold P, Rebmann A, Bhatnagar AS, Evans DB (2006) A single intravenous administration of zoledronic acid prevents the bone loss and mechanical compromise induced by aromatase inhibition in rats. Bone 39:787-795

7. Glatt M, Pataki A, Evans GP, Hornby SB, Green JR (2004) Loss of vertebral bone and mechanical strength in estrogen-deficient rats is prevented by long-term administration of zoledronic acid. Osteoporos Int 15:707-715

8. Glatt M (2001) The bisphosphonate zoledronate prevents vertebral bone loss in mature estrogen-deficient rats as assessed by micro-computed tomography. Eur Cell Mater 1:18-26

9. Hornby SB, Evans GP, Hornby SL, Pataki A, Glatt M, Green JR (2003) Long-term zoledronic acid treatment increases bone structure and mechanical strength of long bones of ovariectomized adult rats. Calcif Tissue Int 72:519-527

10. Brouwers JEM, Lambers JM, Gasser JA, van Rietbergen B, Huiskes R (2008) Bone degeneration and recovery after early and late bisphosphonate treatment of ovariectomized Wistar rats assessed by in vivo micro-computed tomography. Calcif Tissue Int 82:202-211

11. Li M, Shen Y, Qi H, Wronski TJ (1996) Comparative study of skeletal response to estrogen depletion at red and yellow marrow sites in rats. Anat Rec 245:472-480

12. Gasser JA, Ingold P, Venturiere A, Shen V, Green JR (2008) Long-term protective effects of zoledronic acid on cancellous and cortical bone in the ovariectomized rat. J Bone Miner Res 23:544-551

13. Muller R, Ruegsegger P (1997) Micro-tomographic imaging for the nondestructive evaluation of trabecular bone architecture. Stud Health Technol Inform 40:61-79

14. Yao W, Cheng Z, Koester KJ, Ager JW, Balooch M, Pham A, Chefo S, Busse C, Ritchie RO, Lane NE (2007) The degree of bone mineralization is maintained with single intravenous bisphosphonates in aged estrogen-deficient rats and is a strong predictor of bone strength. Bone 41:804-812

15. Li M, Liang H, Shen Y, Wronski TJ (1999) Parathyroid hormone stimulates cancellous bone formation at skeletal sites regardless of marrow composition in ovariectomized rats. Bone 24:95-100

16. Kishi T, Hagino H, Kishimoto H, Nagashima H (1998) Bone responses at various skeletal sites to human parathyroid hormone in ovariectomized rats: effects of long-term administration, withdrawal, and readministration. Bone 22:515-522

17. Miyakoshi N, Sato K, Abe T, Tsuchida T, Tamura Y, Kudo T (1999) Histomorphometric evaluation of the effects of ovariectomy on bone turnover in rat caudal vertebrae. Calcif Tissue Int 64:318-324

18. Ikeda S, Tsurukami H, Ito M, Sakai A, Sakata T, Nishida S, Takeda S, Shiraishi A, Nakamura T (2001) Effect of trabecular bone contour on ultimate strength of lumbar vertebra after bilateral ovariectomy in rats. Bone 28:625-633

19. Gasser JA (2006) The relative merits of anabolics versus antiresorptive compounds: where our targets should be, and whether we are addressing them. Curr Opin Pharmacol 6:313-318

20. Brouwers JEM, van Rietbergen B, Huiskes R (2007) No effects of in vivo micro-CT radiation on structural parameters and bone marrow cells in proximal tibia of Wistar rats detected after eight weekly scans. J Orthop Res 25:1325-1332

21. Recker RR, Delmas PD, Halse J, Reid IR, Boonen S, GarciaHernandez PA, Supronik J, Lewiecki EM, Ochoa L, Miller P, Hu H, Mesenbrink P, Hartl F, Gasser J, Eriksen EF (2008) The effects of intravenous zoledronic acid once yearly on bone remodeling and bone structure. J Bone Miner Res 23:6-16 